\title{
THE AUTOMORPHISM GROUP OF A LIE GROUP
}

\author{
BY \\ G. HOCHSCHILD
}

Introduction. The group $A(G)$ of all continuous and open automorphisms of a locally compact topological group $G$ may be regarded as a topological group, the topology being defined in the usual fashion from the compact and the open subsets of $G$ (see $\$ 1$ ). In general, this topological structure of $A(G)$ is somewhat pathological. For instance, if $G$ is the discretely topologized additive group of an infinite-dimensional vector space over an arbitrary field, then $A(G)$ already fails to be locally compact.

On the other hand, if $G$ is a connected Lie group, we shall show without any difficulty that the compact-open topology of $A(G)$ coincides with the topology obtained by identifying $A(G)$ with a closed subgroup of the linear group of automorphisms of the Lie algebra of $G$, as was done by Chevalley (in [1]) in order to make $A(G)$ into a Lie group. We shall then deduce that $A(G)$ is a Lie group whenever the group of its components, $G / G_{0}$, is finitely generated $\left({ }^{1}\right)$, where $G_{0}$ denotes the component of the identity element in $G$.

The other questions with which we shall be concerned are the following: Let $I\left(G_{0}\right)$ denote the group of the inner automorphisms of $G_{0}$, and let $E\left(G_{0}, G\right)$ denote the natural image in $A\left(G_{0}\right)$ of $A(G)$. Regard $I\left(G_{0}\right)$ and $E\left(G_{0}, G\right)$ as subgroups of $A\left(G_{0}\right)$. Are these subgroups closed in $A\left(G_{0}\right)$ ? Is $E\left(G_{0}, G\right)$ topologically, as well as group-theoretically, isomorphic with the corresponding factor group of $A(G)$ ?

We shall show, under the assumption that $G / G_{0}$ is finitely generated, that these questions are related as follows: The natural continuous homomorphism of $A(G)$ onto $E\left(G_{0}, G\right)$ is open if and only if $E\left(G_{0}, G\right)$ is closed in $A\left(G_{0}\right)$. Under the stronger assumption that $G / G_{0}$ is finite, a sufficient condition for $E\left(G_{0}, G\right)$ to be closed in $A\left(G_{0}\right)$ is that $I\left(G_{0}\right)$ be closed in $A\left(G_{0}\right)$. Finally, in order to throw some light on the difficulties which are involved here, we shall give a simple example in which $I\left(G_{0}\right)$ and $E\left(G_{0}, G\right)$ are not closed in $A\left(G_{0}\right)$. In this example, $G$ has only two components and $G_{0}$ is homeomorphic with Euclidean 5-space.

1. Topological preparation. We shall describe the topology of a group $G$ in terms of a fundamental system $\mathfrak{B}$ of neighborhoods $V$ of the identity element. A system $\mathfrak{B}$ of subsets of $G$ will define a Hausdorff topology consistent with the group operations if and only if it satisfies the following conditions $\left({ }^{2}\right)$ :

Received by the editors May 1, 1951.

(1) I am indebted to the referee for the remark that my original requirement, " $G / G_{0}$ finite", can be relaxed to the present one.

(2) We are taking these from $\$ 2$ of [4]. 
I. The intersection of all $V \in \mathfrak{B}$ is the set consisting of the identity element of $G$ only.

II. If $V_{1}$ and $V_{2}$ are sets belonging to $\mathfrak{B}$, there is a $V \in \mathfrak{B}$ such that $V \subseteq V_{1} \cap V_{2}$.

III. For every $V \in \mathfrak{B}$ there is a $W \in \mathfrak{B}$ such that $W^{-1} W \subseteq V$.

IV. For every $g \in G$ and $V \in \mathfrak{B}$ there is a $W \in \mathfrak{B}$ such that $W \subseteq g V g^{-1}$.

The neighborhoods of the identity element are then all the sets containing a set belonging to $\mathfrak{B}$.

If $C$ is any compact subset of $G$ and $V \in \mathfrak{B}$, we denote by $N(C, V)$ the set of all $\alpha \in A(G)$ for which $\alpha(x) x^{-1} \in V$ and $\alpha^{-1}(x) x^{-1} \in V$, whenever $x \in C$. We claim that if $G$ is locally compact, the system of these $N(C, V)$ satisfies conditions I-IV above. In fact, I holds quite evidently. If $V \subseteq V_{1} \cap V_{2}$, we clearly have $N\left(C_{1} \cup C_{2}, V\right) \subseteq N\left(C_{1}, V_{1}\right) \cap N\left(C_{2}, V_{2}\right)$, so that II is satisfied. In order to verify III we proceed as follows: Since $G$ is locally compact, given $V \in \mathfrak{B}$, there is a compact set $C_{0}$ and a $V_{0} \in \mathfrak{B}$ such that $V_{0} \subseteq C_{0}$ and $V_{0} V_{0} V_{0} \subseteq V$. From the identity $\left(\beta^{-1} \alpha\right)(c) c^{-1}=\left[\beta^{-1}\left(\alpha(c) c^{-1}\right)\left(\alpha(c) c^{-1}\right)^{-1}\right]$ - $\left[\alpha(c) c^{-1}\right]\left[\beta^{-1}(c) c^{-1}\right]$ we can see immediately that we have then $N\left(C \cup C_{0}, V_{0}\right)^{-1} N\left(C \cup C_{0}, V_{0}\right) \subseteq N(C, V)$, which shows that III is satisfied. Finally, we have, with $\alpha \in A(G), \alpha^{-1} N(\alpha(C), \alpha(V)) \alpha \subseteq N(C, V)$, whence IV holds.

From now on, if $G$ is any locally compact group, $A(G)$ will denote the group of all continuous and open automorphisms of $G$, with the topology defined by the $N(C, V)$, where $C$ ranges over the compact subsets of $G$ and $V$ over the set of neighborhoods of the identity element in $G$.

Next we shall prove two elementary results which we shall need later on.

Lemma 1. Let $G$ be a topological group, $U$ a neighborhood of the identity element, $C$ a compact subset of $G$. Then the intersection of all the sets $c^{-1} U c$, with $c \in C$, is a neighborhood of the identity element.

Proof. We can find $V \in \mathfrak{B}$ such that $V V V^{-1} \subseteq U$. Then we have $y^{-1} U y$ $\supseteq V$, for every $y \in V$. Since $C$ is compact, there are elements $c_{1}, \cdots, c_{n}$ in $C$ such that $C$ is contained in the union of the $n$ sets $V c_{i}$. Now if $c$ is any element in $C$, we write $c=y c_{i}$, with $y \in V$. Then $c^{-1} U c=c_{i}^{-1} y^{-1} U y c_{i} \supseteq c_{i}^{-1} V c_{i}$. Hence the intersection of all the sets $c^{-1} U c$ contains the finite intersection of the $c_{i}^{-1} V c_{i}$ and is therefore a neighborhood of the identity element.

Lemma 2. Suppose that $G$ is connected and locally compact. Let $C$ be a compact subset of $G, V$ a neighborhood of the identity, and $S$ a compact neighborhood of the identity. Then there exists a neighborhood $W$ of the identity such that $N(S, W) \subseteq N(C, V)$.

Proof. Since $G$ is connected and $S$ a neighborhood of the identity, we have $G=\cup_{n=1}^{\infty} S^{n}$. Since $C$ is compact and since $S^{n} \subseteq S^{n+1}$, it follows that $C \subseteq S^{m}$, for some $m$. Now choose a neighborhood $T$ of the identity such that $T^{m} \subseteq V$, 
and put $W=\bigcap_{x \in s^{m}} x^{-1} T x$. By Lemma $1, W$ is a neighborhood of the identity, for $S^{m}$ is compact $\left.{ }^{3}\right)$. Now let $\alpha \in N(S, W)$ and $c \in C$. We have $c=x_{1}, \cdots, x_{m}$, with $x_{i} \in S$. Put $c_{k}=x_{1} \cdots x_{k}$, and suppose we have already shown that $\alpha\left(c_{k}\right) c_{\mathbf{k}}^{-1} \in T^{k}$. Then we have $\alpha\left(c_{k+1}\right) c_{\mathbf{k}_{+1}}^{-1}=\left(\alpha\left(c_{k}\right) c_{\mathbf{k}}^{-1}\right) c_{k}\left(\alpha\left(x_{k+1}\right) x_{\mathbf{k}+1}^{-1}\right) c_{\mathbf{k}}^{-1}$ $\in T^{k} c_{k} W c_{\mathbf{k}}^{-1} \subseteq T^{k+1}$. Hence we get $\alpha(c) c^{-1} \in T^{m} \subseteq V$, which clearly suffices to establish our lemma.

2. Automorphism groups. Let $G$ be a connected Lie group. An automorphism $\alpha \in A(G)$ induces an automorphism $\dot{\alpha}$ of the Lie algebra \&s of $G$. We denote by $A(B)$ the group of automorphisms of $B$, with the topology induced by that of the full linear group of which $A(\mathbb{B})$ is clearly a closed subgroup. It is shown in [1] that the mapping $\alpha \rightarrow \dot{\alpha}$ is a group isomorphism of $A(G)$ onto a closed subgroup of $A$ (S)). (If $G$ is simply-connected the image of of $A(G)$ coincides with $A(\mathbb{S})$.) We shall prove the following result:

TheOREM 1. Let $G$ be a connected Lie group. Then the group isomorphism $\alpha \rightarrow \dot{\alpha}$ of $A(G)$ onto the corresponding closed subgroup of $A(\mathfrak{G})$ is also a homeomorphism.

Proof. We denote by $e$ the "exponential mapping" of \& into $G\left({ }^{4}\right)$. We have then, for $\alpha \in A(G), e \dot{\alpha}=\alpha e$, and $e$ gives an analytic isomorphism between a neighborhood of 0 in $\&$ and a neighborhood of the identity in $G$. Let $z_{1}, \cdots, z_{n}$ be a linear basis for $(\$)$ such that the corresponding solid sphere $3_{2}$ of radius 2 , in the Euclidean metric defined by our basis, around 0 in $(\$)$, is mapped by $e$ 1-1 and analytically on to the canonical sphere $Z_{2}=e\left(\Omega_{2}\right)$ around the identity element in $G$. For any positive real number $p, Z_{p}$ will denote the closed solid sphere of radius $p$ around 0 in $\left(S\right.$, and we set $Z_{p}=e\left(B_{p}\right)$.

Now if $N$ is any neighborhood of the identity in $A(\mathfrak{s})$, there is a real number $s$ such that $0<s<1$ and such that every $\tau \in A(\mathbb{S})$ satisfying $\tau\left(z_{i}\right)$ $-z_{i} \in Z_{8}$ belongs to $N$. It follows from the elementary properties of the exponential mapping $e$ that there is a real number $q>1$ and a real number $r$, $s>r>0$, such that, for all $a, b \in Z_{r}$, we have $e(a) e(b)=e(a+b+c)$, with $|c|$ $<q \cdot|a| \cdot|b|$, where $|u|$ denotes the distance of $u$ from 0 in (5). Let $\alpha$ $\in N\left(Z_{r}, Z_{r^{2} / 2 q}\right)$. Then, for $0 \leqq t \leqq r, \alpha\left(e\left(t z_{i}\right)\right) e\left(t z_{i}\right)^{-1}=e\left(u_{i}(t)\right)$, where $u_{i}(t)$ $\in B^{2} / 2 q$. Hence $e\left(t \dot{\alpha}\left(z_{i}\right)\right)=e\left(u_{i}(t)\right) e\left(t z_{i}\right)=e\left(u_{i}(t)+t z_{i}+v_{i}(t)\right)$, with $\left|v_{i}(t)\right|$ $<q\left|u_{i}(t)\right| t \leqq r^{3} / 2$. In particular, this shows that $t \dot{\alpha}\left(z_{i}\right)$ remains in $B_{2 r}$ as $t$ varies from 0 to $r$, and that we must have $r\left(\dot{\alpha}\left(z_{i}\right)-z_{i}\right)=u_{i}(r)+v_{i}(r)$. Therefore, $\left|\ddot{\alpha}\left(z_{i}\right)-z_{i}\right| \leqq r / 2 q+r^{2} / 2<r<s$, whence $\ddot{\alpha} \in N$. Thus we have shown that $N\left(Z_{r}, Z_{r^{2} / 2 q}\right)^{\circ} \subseteq N$, and this implies that the mapping $\alpha \rightarrow \dot{\alpha}$ is continuous.

On the other hand, given any neighborhood $V$ of the identity in $G$, we can find a real number $r>0$ such that $e(u+z) \in V e(z)$ whenever $|z| \leqq 1$ and $|u| \leqq r$, because $e$ is uniformly continuous on $Z_{2}$. Hence if $\dot{\alpha}$ is such that $\dot{\alpha}(z)-z \in Z_{r}$ for all $z \in Z_{1}$, then $\alpha(x) x^{-1} \in V$ for all $x \in Z_{1}$. Since the $N\left(Z_{1}, V\right)$

(3) If $A$ and $B$ are compact, so is $A B$; see $\$ 3$ of [4].

(4) See §VIII, Chap. IV, of [1]. 
constitute a fundamental system of neighborhoods of the identity in $A(G)$, by Lemma 2 , and since the $\dot{\alpha}$ satisfying the above conditions make up a neighborhood of the identity in the image of $A(G)$ in $A(\mathbb{S})$, we conclude that the mapping $\dot{\alpha} \rightarrow \alpha$ is also continuous. This completes the proof of Theorem 1.

Theorem 2. Let $G$ be a Lie group, and let $G_{0}$ denote the component of the identity in $G$. Suppose that the group of components, $G / G_{0}$, is finitely generated. Then $A(G)$ is a Lie group and has at most countably many components.

Proof. Our assumption on $G / G_{0}$ means that there is a finite set $g_{1}, \cdots, g_{m}$ of elements of $G$ such that every component $P$ of $G$ is of the form $P=p G_{0}$, where $p$ is a product of $g_{i}$ 's (with repetitions allowed). Now let $B$ denote the subgroup of $A(G)$ which consists of all those automorphisms that map each component of $G$ onto itself. It is clear from the form $p G_{0}$ of a component that we have $N\left(\left(g_{1}, \cdots, g_{m}\right), G_{0}\right) \subseteq B$, whence we see that $B$ is open in $A(G)$. (Note that $G_{0}$ is open in $G$.) Furthermore, since $A(G) / B$ is isomorphic with a subgroup of the automorphism group $A\left(G / G_{0}\right)$ of the finitely generated group $G / G_{0}$, it follows that $A(G) / B$ has at most countably many elements. Hence it will suffice to prove that $B$ is a Lie group with at most countably many components.

Let $H$ be the semi-direct product $\left(G_{0}^{m} \times A\left(G_{0}\right)\right)_{A}$ whose elements are the $m+1$-tuples $\left(c_{1}, \cdots, c_{m}, \alpha\right)$, with $c_{i} \in G_{0}$ and $\alpha \in A\left(G_{0}\right)$, and where products are defined by the formula $\left(c_{1}, \cdots, c_{m}, \alpha\right)\left(d_{1}, \cdots, d_{m}, \beta\right)=\left(c_{1} \alpha\left(d_{1}\right), \cdots\right.$, $\left.c_{m} \alpha\left(d_{m}\right), \alpha \beta\right)$. If we topologize $H$ by the natural product topology it is evident, since $A\left(G_{0}\right)$ is a Lie group, that $H$ is a Lie group. Furthermore, since $A\left(G_{0}\right)$ may, according to Theorem 1 , be identified with a subgroup of the full linear group, its topology satisfies the second axiom of countability, and the same holds therefore for $H$.

Now we define a mapping $\phi$ of $B$ into $H$ by setting $\phi(b)=\left(g_{1}^{-1} b\left(g_{1}\right), \cdots\right.$, $\left.g_{m}^{-1} b\left(g_{m}\right), \beta\right)$, where $\beta$ is the restriction of $b$ to $G_{0}$. It is immediately seen that $\phi$ is a continuous isomorphism of $B$ into $H$. We show next that $\phi^{-1}$ maps $\phi(B)$ continuously onto $B$. For this it suffices to show that, if $V$ is any neighborhood of the identity in $G$ and $C$ any compact subset of $G$, there is a neighborhood $M$ of the identity in $H$ for which $\phi^{-1}(M \cap \phi(B)) \subseteq N(C, V)$. Now, since $C$ is compact, there is a finite set $p_{1}, \cdots, p_{k}$ of products of the $g_{i}$ 's such that $C \subseteq \bigcup_{j=1}^{k} p_{j} G_{0}$. Let $S=\bigcup_{j=1}^{k}\left(p_{j}^{-1} C\right) \cap G_{0}$, and let $W$ be a neighborhood of the identity in $G_{0}$. Note that $S$ is compact, so that the corresponding subset $N_{0}(S, W)$ of $A\left(G_{0}\right)$ is a neighborhood of the identity in $A\left(G_{0}\right)$. Put $U$ $=W \times \cdots \times W \times N_{0}(S, W)$. Then $U$ is a neighborhood of the identity in $H$, and so is $M=U \cap U^{-1}$. Since every element of $C$ can be written in the form $c=p_{j} s$, with $s \in S$, it is not hard to see that, with a suitable choice of $W$, the neighborhood $M$ will satisfy our above requirement. Hence $\phi$ is a homeomorphism of $B$ onto $\phi(B)$. 
Now for each component $P$ of $G$ select an explicit product $p$ of $g_{i}$ 's such that $P=p G$. Let $\eta=\left(c_{1}, \cdots, c_{m}, \alpha\right)$ be an arbitrary element $H$. Let $p^{\prime}$ denote the element of $G$ which is obtained from $p$ by replacing each $g_{i}$ by $g_{i} c_{i}$. For $x \in G_{0}$, define $\tilde{\eta}(p x)=p^{\prime} \alpha(x)$. Then $\tilde{\eta}$ is evidently a homeomorphism of $G$ onto itself. It follows that $\eta$ will belong to $\phi(B)$ if and only if $\tilde{\eta}(u v)=\tilde{\eta}(u) \tilde{\eta}(v)$, for all $u, v \in G$. This shows that $\phi(B)$ is closed in $H$. Hence $\phi(B)$ is a Lie group. Since $H$ satisfies the second axiom of countability, so does $\phi(B)$. Since the components of a Lie group are open sets, it follows that the number of components of $B$ is at most countable. Hence also $B$ is a Lie group with at most countably many components, and our proof is complete.

3. The restriction homomorphism. Let $G$ be a Lie group, $G_{0}$ the component of the identity element in $G$. We assume that $G / G_{0}$ is finitely generated, or-which is equivalent-that $G$ is generated by a compact subset. The restriction of automorphisms to $G_{0}$ evidently gives a continuous homomorphism, $\rho$ say, of $A(G)$ onto a subgroup $E\left(G_{0}, G\right)$ of $A\left(G_{0}\right)$. Let $R$ denote the kernel of $\rho$. It is natural to inquire under what conditions $A(G) / R$ is isomorphic, as a topological group, with $E\left(G_{0}, G\right)$, or, equivalently, under what conditions $\rho$ is open. A superficial answer is given by the following theorem:

THEOREM 3. The restriction homomorphism $\rho$ of $A(G)$ onto $E\left(G_{0}, G\right)$ is open if and only if $E\left(G_{0}, G\right)$ is closed in $A\left(G_{0}\right)$.

Proof. Suppose first that $\rho$ is open. Then $E\left(G_{0}, G\right)$ is homeomorphic with $A(G) / R$ and hence is locally compact. It follows from this, as is well known, that $E\left(G_{0}, G\right)$ is closed in $\left.A\left(G_{0}\right){ }^{5}\right)$. Conversely, if $E\left(G_{0}, G\right)$ is closed in $A\left(G_{0}\right)$, then it is locally compact. Furthermore, as a subspace of $A\left(G_{0}\right)$, it satisfies the second axiom of countability. By Theorem $2, A(G)$ is locally compact and satisfies the second axiom of countability. By a well known result $\left({ }^{6}\right)$, the continuous homomorphism $\rho$ must therefore be open. This completes the proof.

We shall now give an example in which $E\left(G_{0}, G\right)$ is not closed in $A\left(G_{0}\right)$ : Let $C$ denote the additive group of the complex numbers, $R$ the additive group of the real numbers, both with the ordinary topology. We form the semi-direct product $G_{0}=(C \times C \times R)_{h}$ with the multiplication

$$
\left(c_{1}, c_{2}, r\right)\left(c_{1}^{\prime}, c_{2}^{\prime}, r^{\prime}\right)=\left(c_{1}+e^{2 \pi i r} c_{1}^{\prime}, c_{2}+e^{2 \pi i h r} c_{2}^{\prime}, r+r^{\prime}\right) \text {, }
$$

where $h$ is a fixed irrational real number. Evidently, $G_{0}$ is a Lie group, and its underlying space is Euclidean 5-space.

Next we construct a semi-direct product $G$ of $G_{0}$ by a group of order 2, with a generator $g$ such that $g^{2}$ is the identity element $(0,0,0)$ of $G_{0}$ and $G$,

(5) See $\S 3$ of [4].

(6) Theorem 13, chap. III, in [3]. 
and $g\left(c_{1}, c_{2}, r\right) g^{-1}=\gamma\left(c_{1}, c_{2}, r\right)=\left(\bar{c}_{1}, \bar{c}_{2},-r\right)$, where $\bar{c}$ denotes the complex conjugate of $c$.

With $s, t$ arbitrary real numbers, let $\alpha=\alpha_{s, t}$ be the automorphism of $G_{0}$ which is defined by setting $\alpha\left(c_{1}, c_{2}, r\right)=\left(e^{2 \pi i s} c_{1}, e^{2 \pi i t} c_{2}, r\right)$. We shall determine the extensions of $\alpha$ to $G$. Let $\tilde{\alpha} \in A(G)$ be such that $\rho(\tilde{\alpha})=\alpha$. We must have $\tilde{\alpha}(g)=g z$, with $z \in G_{0}$. If we write down the equations which express the fact that $\tilde{\alpha}$ is a homomorphism, we find that we must have: (1) $\gamma(z) z=(0,0,0)$, and (2) $z \alpha \gamma(x)=\gamma \alpha(x) z$, for all $x \in G_{0}$.

Conversely, every element $z \in G_{0}$ which satisfies these conditions defines an extension $\tilde{\alpha} \in A(G)$ of $\alpha$.

Write $z=\left(c_{1}, c_{2}, r\right)$. If we write down conditions (2) with $x=(0,0, u)$, we find that we must have $c_{1}=0=c_{2}$. Then condition (1) holds with arbitrary $r \in R$. If we rewrite conditions (2) with $z=(0,0, r)$ and all $x \in G_{0}$, we find that they are equivalent to the condition that $r+2 s$ and $h r+2 t$ be integers. Now, since $h$ is irrational, we can find a sequence of integers $k_{n}$ such that the congruence class $\bmod 1$ of $h k_{n} / 2$ approaches the congruence class of $1 / 3$ as $n$ becomes large. Put $s_{n}=1 / 2 h n$ and $t_{n}=1 / 2 n+h k_{n} / 2$. Let $\alpha_{n}=\alpha_{s_{n}}, t_{n}$. If $r_{n}=-k_{n}$ $-1 / h n$, then it satisfies our above conditions on $r$, whence we conclude that each $\alpha_{n}$ belongs to $E\left(G_{0}, G\right)$. On the other hand, $\alpha_{n}$ evidently approaches the automorphism $\alpha_{0,1 / 3}$ in $A\left(G_{0}\right)$, and we claim that $\alpha_{0,1 / 3} \notin E\left(G_{0}, G\right)$. In fact, the conditions for the number $r$ needed for extending $\alpha_{0,1 / 3}$ become $r=m$, and $h r=n-2 / 3$, where $m$ and $n$ are integers. But these conditions are incompatible, because $h$ is irrational. Hence $E\left(G_{0}, G\right)$ is not closed in $A\left(G_{0}\right)$.

It will be apparent from the next theorem that in the above example the group $I\left(G_{0}\right)$ of the inner automorphisms of $G_{0}$ is not closed in $A\left(G_{0}\right)$; a fact which could also be shown quite directly by considering the above automorphisms $\alpha_{8, t}$.

THEOREM 4. If $G / G_{0}$ is finite and $I\left(G_{0}\right)$ is closed in $A\left(G_{0}\right)$, then the restriction homomorphism $\rho$ of $A(G)$ onto $E\left(G_{0}, G\right)$ is open.

Proof. Let $B$ denote the subgroup of $A(G)$ whose elements map each component $g_{i} G_{0}, i=1, \cdots, m$, of $G$ onto itself. We claim that it suffices to show that the restriction of $\rho$ to $B$ is an open homomorphism of $B$ onto $\rho(B)$. In fact, if this has been proved, we may conclude that $\rho(B)$ is homeomorphic with $B / R \cap B$, where $R$ is the kernel of $\rho$, and hence that $\rho(B)$ is locally compact. This implies that $\rho(B)$ is closed in $E\left(G_{0}, G\right)$. Since $A(G) / B$ is finite, so is $E\left(G_{0}, G\right) / \rho(B)$. Hence the complement of $\rho(B)$ in $E\left(G_{0}, G\right)$ is the union of a finite number of cosets of $\rho(B)$ and hence is closed. Hence $\rho(B)$ is open in $E\left(G_{0}, G\right)$. It follows that $\rho$ is an open homomorphism of $A(G)$ onto $E\left(G_{0}, G\right)$, which proves our claim.

Now let us observe that our assumption on $I\left(G_{0}\right)$ implies that the natural homomorphism of $G_{0}$ onto $I\left(G_{0}\right)$ is open, as well as continuous. Indeed, the continuity is independent of our assumption; for, given a compact subset 
$C$ of $G_{0}$ and a neighborhood $V$ of the identity in $G_{0}$, it is clear that for each $c \in C$ we can find a neighborhood $V_{c}$ of the identity such that $u x u^{-1} x^{-1} \in V$ for all $u \in V_{c}$ and $x \in c V_{c}$. Since $C$ is compact, there is a finite subset $c_{1}, \cdots$, $c_{q}$ of $C$ such that $C \subseteq \bigcup_{i=1}^{q} c_{i} V_{c_{i}}$. Then, if $W=\bigcap_{i=1}^{q} V_{c_{i}}$, we have $u x u^{-1} x^{-1} \in V$, for all $u \in W$ and $x \in C$, which proves that the natural homomorphism of $G_{0}$ onto $I\left(G_{0}\right)$ is always continuous. Now if $I\left(G_{0}\right)$ is closed in $A\left(G_{0}\right)$, then it is a connected Lie group. Since $G_{0}$ is also a connected Lie group, the continuous natural homomorphism of $G_{0}$ onto $I\left(G_{0}\right)$ must automatically be open.

It will be convenient to identify $B$ with the closed subgroup $\phi(B)$ of the group $H$ which we introduced in the proof of Theorem 2. It is easy to check that $\phi(R \cap B)$ is precisely the set of elements $\left(z_{1}, \cdots, z_{m}, 1\right) \in H$, where 1 stands for the identity automorphism of $G_{0}$, and where the elements $z_{i}$ belong to the center, $Z_{0}$, say, of $G_{0}$ and satisfy the relations $g_{j}^{-1} z_{i} g_{j} z_{j}=z_{k(i, j)}$, the index $k(i, j)$ being determined by the relation $g_{i} g_{j} \in g_{k(i, j)} G_{0}$.

What we still have to prove therefore amounts to the following: Given a neighborhood $U$ of the identity in $G_{0}$, there is a neighborhood $M$ of the identity in $A\left(G_{0}\right)$, such that, for every element $\left(c_{1}, \cdots, c_{m}, \alpha\right) \in \phi(B)$ with $\alpha \in M$, we can find elements $z_{i}$ as above and such that $z_{i} c_{i} \in U\left({ }^{7}\right)$.

Since $Z_{0}$ is a Lie group, there is a neighborhood $D$ of the identity in $G_{0}$ which has the following properties:

(1) For every $z \in D \cap Z_{0}$, there is an element $t \in D \cap Z_{0}$ such that $t^{m}=z$.

(2) If $s, t$ belong to $\left(g_{j}^{-1} D g_{j} D D^{-1}\right) \cap Z_{0}$ and $s^{m}=t^{m}$, then $s=t$.

(3) $D D \subseteq U$.

Let us choose a neighborhhod $S$ of the identity in $G_{0}$ such that $S^{2 m} \subseteq D$. Let $\gamma_{i}$ denote the inner automorphism $u \rightarrow g_{i}^{-1} u g_{i}$, and choose a neighborhood $V$ of the identity in $G_{0}$ such that $V=V^{-1}$ and $V \gamma_{i} \gamma_{j} \gamma_{k}^{-1}(V V) \subseteq S$, for all $i, j, k$.

Now observe that if $\phi(b)=\left(c_{1}, \cdots, c_{m}, \alpha\right)$, then $\gamma_{i} \alpha \gamma_{i}^{-1} \alpha^{-1}(x)=c_{i} x c_{i}^{-1}$, for every $x \in G_{0}$. Since the homomorphism of $G_{0}$ onto $I\left(G_{0}\right)$ is open, we can find a neighborhood $M^{\prime}$ of the identity in $A\left(G_{0}\right)$ such that every automorphism belonging to $I\left(G_{0}\right) \cap M^{\prime}$ is effected by an element belonging to $V$. Choose a neighborhood $M$ of the identity in $A\left(G_{0}\right)$ such that, for every $\alpha \in M$, we have $\gamma_{i} \alpha \gamma_{i}^{-1} \alpha^{-1} \in M^{\prime}$, for each $i$, and also $\alpha(a(i, j)) a(i, j)^{-1} \in V$, for each pair $(i, j)$, where $a(i, j)=g_{k(i, j)}^{-1} g_{i} g_{j}$.

Now let $\alpha \in M$ and $\left(c_{1}, \cdots, c_{m}, \alpha\right)=\phi(b)$. Then the inner automorphism of $G_{0}$ which is effected by $c_{i}$ is also effected by an element $v_{i} \in V$, whence $c_{i}=t_{i} v_{i}$, with $t_{i} \in Z_{0}$. We have $\gamma_{j}\left(c_{i}\right) c_{j}=g_{j}^{-1} g_{i}^{-1} b\left(g_{i} g_{j}\right)=g_{j}^{-1} g_{i}^{-1} g_{k(i, j)} c_{k(i, j)} \alpha(a(i, j))$

(7) For the argument which now follows I am indebted to T. Nakayama. The idea of this proof is that if $\alpha$ is "small" enough the $c_{i}$ can be replaced by "small" elements. This possibility is due to the fact that a factor set, defined on $G / G_{0}$, and with sufficiently small values in $Z_{0}$, must be a transformation set, because unique divisibility holds near the identity in $Z_{0}$. Such a device has been used by Iwasawa on p. 510 of [2] in dealing with the automorphisms of a compact group. 
$=a(i, j)^{-1} c_{k(i, j)} \alpha(a(i, j))$, whence $\gamma_{j}\left(t_{i}\right) t_{j} t_{k(i, j)}^{-1}=a(i, j)^{-1} v_{k(i, j)} \alpha(a(i, j)) v_{j}^{-1} \gamma_{j}\left(v_{i}^{-1}\right)$ $=\gamma_{j} \gamma_{i} \gamma_{k}^{-1}{ }_{(i, j)}\left(v_{k(i, j)} \alpha(a(i, j)) a(i, j)^{-1}\right) v_{j}^{-1} \gamma_{j}\left(v_{i}^{-1}\right) \in S^{2}$. Hence $\prod_{i=1}^{m} \gamma_{j}\left(t_{i}\right) t_{j} t_{k}^{-1}{ }_{(i, j)}$ $\in S^{2 m} \subseteq D$, i.e., $\gamma_{j}(t) t^{-1} t_{j}^{m} \in D \cap Z_{0}$, where $t=t_{1} \cdots t_{m}$. By property (1) of $D$, there are elements $u_{j} \in D \cap Z_{0}$ such that $u_{j}^{m}=\gamma_{j}(t) t^{-1} t_{j}^{m}$, and then we have $\left(\gamma_{j}\left(u_{i}\right) u_{j} u_{k(1, j)}^{-1}\right)^{m}=\left(\gamma_{j}\left(t_{i}\right) t_{j} t_{k(1, j)}^{-1}\right)^{m}$. By property (2) of $D$, it follows that $\gamma_{j}\left(u_{i}\right) u_{j} u_{k(i, j)}^{-1}=\gamma_{j}\left(t_{i}\right) t_{j} t_{k(i, j)}^{-1}$. Set $z_{i}=u_{i} t_{i}^{-1}$. Then $g_{j}^{-1} z_{i} g_{j} z_{j}=z_{k(i, j)}$, and $z_{i} c_{i}=u_{i} v_{i}$ $\in D D \subseteq U$. This is what we had to prove in order to establish Theorem 4.

CoRollary. If $G_{0} / Z_{0}$ is compact, or if $G_{0}$ is semisimple, then the restriction homomorphism of $A(G)$ onto $E\left(G_{0}, G\right)$ is open.

Proof. If $G_{0} / Z_{0}$ is compact, so is $I\left(G_{0}\right)$, and hence $I\left(G_{0}\right)$ is closed in $A\left(G_{0}\right)$. If $G_{0}$ is semisimple, then $I\left(G_{0}\right)$ coincides with the component of the identity in $A\left(G_{0}\right)$, and hence is closed in $A\left(G_{0}\right)$.

\section{BIBLIOGRAPHY}

1. C. Chevalley, Theory of Lie groups, Princeton, 1946. 557.

2. K. Iwasawa, On some types of topological groups, Ann. of Math. vol. 50 (1949) pp. 507-

3. L. Pontrjagin, Topological groups, Princeton, 1939.

4. A. Weil, L'integration dans les groupes topologiques et ses applications, Paris, 1938.

UNIVERSITY OF ILLINOIS,

URBANA, ILL. 